\title{
Performance of a highly sensitive optical waveguide Mach-Zehnder interferometer immunosensor
}

\author{
R. G. Heideman, R. P. H. Kooyman and J. Greve \\ Mesa Research Institute, Department of Applied Physics, University of Twente, P.O. Box 217, 7500 AE Enschede (Netherlands)
}

(Received February 10, 1992; in revised form August 6, 1992; accepted August 8, 1992)

\begin{abstract}
We describe a highly sensitive sensor which uses the evanescent field of a reusable planar optical waveguide as the sensing element. The waveguide used is optimized to obtain a steep dependence of the propagation velocity on the refractive-index profile near the surface. The adsorption of a layer of proteins thus results in a phase change, which is measured interferometrically using a Mach-Zehnder interferometer set-up. The stability of the interferometer is such that phase changes $\approx\left(1 \times 10^{-2}\right) 2 \pi$ per hour can be measured. Immunoreactions have been monitored down to concentrations of $5 \times 10^{-11} \mathrm{M}$ of a $40 \mathrm{kDa}$ protein.
\end{abstract}

\section{Introduction}

The evanescent field built up in an optical waveguide structure can be used to obtain sensitive immunosensors [1], because of the opportunity of using long interaction lengths. The procedure is to immobilize the receptor molecule (the antibody) onto the waveguide surface, where a specific reaction with its antigen (the analyte) results in the growth of an additional layer. This causes a changing refractive-index profile in the vicinity of the waveguide surface, within the evanescentfield region. The resulting change of the waveguide configuration can be measured by a variety of optical methods [2].

By using an interferometric set-up, the change in the propagation velocity of the light passing through the waveguide is detected. The Mach-Zehnder set-up seems to be the most adequate interferometer configuration for our purposes, because the presence of an easily accessible reference arm allows for compensation of common-mode effects, such as temperature variations.

The combination of interferometric detection and relatively long interaction lengths gives rise to a potentially very sensitive sensor $[3,4]$, which can be used to monitor various kinds of surface processes that result in a changing refractive-index profile within the penetration depth of the evanescent field of the optical waveguide. In this communication we demonstrate the use of such a waveguide interferometer as an immunosensor with picomolar sensitivity.

\section{Sensitivity calculations}

The sensing principle is the changing waveguide configuration as a result of a changing refractive-index profile within the evanescent field of the mode propagating through the waveguide, which is measured with an interferometer, see Fig. 1. This means that the sensor is not only sensitive to surface reactions, but also to

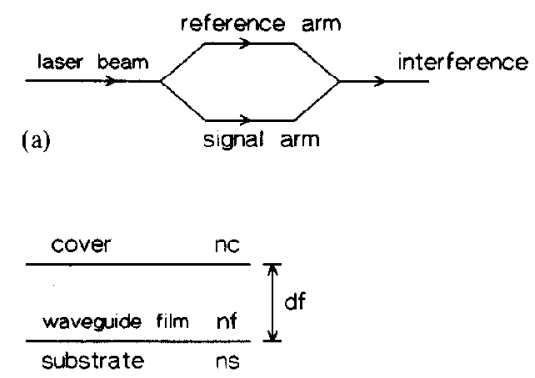

(b)

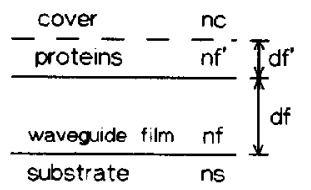

(c)

Fig. 1. General interferometric set-up (a) and the planar waveguide before (b) and after (c) protein adsorption at the surface. The effective refractive index $N$ of the waveguide configuration is changed accordingly. 
bulk refractive-index changes. In the following we shall concentrate on the optical phase change $\Delta \phi$ that results from a change in the refractive-index profile at the waveguide surface. It is this quantity that is measured in a Mach-Zehnder set-up. Assuming small changes $\Delta n_{\mathrm{c}, \text { eff }}$ we obtain

$\Delta \phi=L\left(2 \pi / \lambda_{0}\right)\left(\frac{\partial N}{\partial n_{\mathrm{c}, \mathrm{eff}}}\right) \Delta n_{\mathrm{c}, \mathrm{eff}}$

with $L=$ interaction length, $\lambda_{0}=$ wavelength of the laser light used, $N=$ effective refractive index of the mode and $\Delta n_{\mathrm{c} \text {, eff }}=$ change in the effective refractive index of the cover, including surface reactions.

The parameter $n_{\mathrm{c}, \text { eff }}$ is the effective refractive index of the cover, defined by [14]

$n_{\mathrm{c}, \mathrm{eff}}^{2}=\frac{1}{z_{0}^{\prime}} \int_{0}^{\infty} \exp \left(-z / z_{0}^{\prime}\right) n^{2}(z) \mathrm{d} z$

with $z_{0}^{\prime}=z_{0} / 2, z_{0}$ is the evanescent penetration depth and $n(z)$ is the refractive-index profile perpendicular to the surface of the waveguide.

Now we assume that $n(z)$ can be approximated by a step profile, $n\left(z<d_{f}^{\prime}\right)=n_{\mathrm{f}}^{\prime}$ and $n\left(z>d_{\mathrm{f}}^{\prime}\right)=n_{\mathrm{c}}$, corresponding to a situation where a homogeneous protein layer is adsorbed, with thickness $d_{\mathrm{f}}^{\prime}$ and refractive index $n_{f}^{\prime}$. Here $n_{f}^{\prime}$ is assumed to be constant during the biochemical reactions. This implies that the layer does not change its structure during the binding process.

Equation (2) can then be rewritten as

$$
n_{\mathrm{c}, \text { eff }}^{2}=\frac{d_{\mathrm{f}}^{\prime \prime}}{z_{0}^{\prime}}\left(n_{\mathrm{f}}^{\prime 2}-n_{\mathrm{c}}^{2}\right)+n_{\mathrm{c}}^{2}
$$

Thus, in good approximation, for the case when $d_{\mathrm{f}}^{\prime} \ll z_{0}$ and $n_{\mathrm{f}}^{\prime}$ is constant, eqn. (1a) transforms to

$\Delta \phi=L\left(2 \pi / \lambda_{0}\right)\left(\frac{\partial N}{\partial n_{\mathrm{c}}} \Delta n_{\mathrm{c}}+\frac{\partial N}{\partial d_{\mathrm{f}}^{\prime}} \Delta d_{\mathrm{f}}^{\prime}\right)$

It can be calculated $[3,5]$ that the sensitivities $\partial N / \partial n_{\mathrm{c}}$ and $\partial N / \partial d_{\mathrm{f}}^{\prime}$ result in slightly different optimum waveguide thicknesses. This difference, however, is not sufficient for a substantial suppression of the sensor's bulk refractive-index sensitivity. Thus the insensitivity to bulk changes has to be accomplished completely by matching the optical paths in both arms.

In order to obtain the maximum sensitivity for biochemical surface reactions, it is clear from eqn. (1b) that, apart from increasing the interaction length $L$, $\partial N / \partial d_{f}^{\prime}$ should be as large as possible. It can be shown [5] that this is achieved by:

(a) maximizing the difference between the refractive indices of the actual waveguiding layer $\left(n_{\mathrm{f}}\right)$ and the substrate $\left(n_{\mathrm{s}}\right)$ :

$n_{\mathrm{r}} \gg n_{\mathrm{s}}$

(b) adjusting the thickness of the waveguiding layer $\left(d_{\mathrm{f}}\right)$ to be approximately twice the cut-off thickness:

$d_{\mathrm{f}} \approx 2 d_{\text {cut-off }}$

In Fig. 2 this sensitivity behaviour is shown by plotting the effective refractive index against the waveguide thickness, with $n_{\mathrm{f}}$ as parameter. In this plot we assumed a substrate index $n_{\mathrm{s}}=1.457\left(\mathrm{SiO}_{2}\right)$, and a cover index $n_{\mathrm{c}}=1.33$ (water) (see Section 3 ). In agreement with previous results [4], the highest sensitivity is obtained where the tangent at the curves is maximal

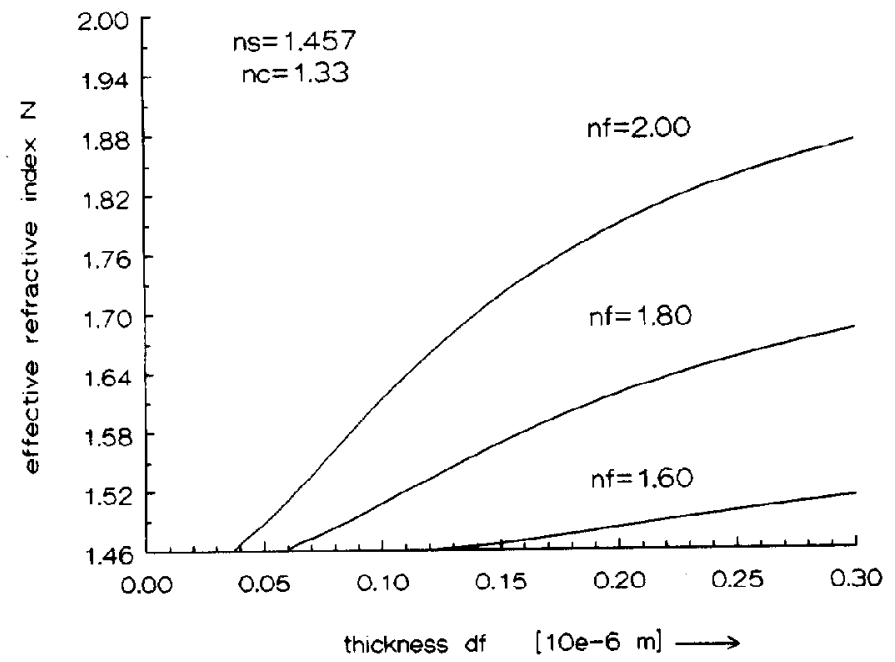

Fig. 2. Plot of the effective refractive index $N$ vs. the thickness of the waveguiding layer $d_{f}$, with refractive index $n_{\mathrm{f}}$ as parameter. 
(because a growth of $d_{\mathrm{f}}^{\prime}$ can be regarded as a change in $d_{\mathrm{f}}$ ).

Besides the sensitivity considerations, several limiting conditions apply to the waveguide structure. First, it is evident that the configuration should have good waveguiding characteristics. Furthermore, to obtain replicable structures and the possibility of mass-production, the waveguide structure should be compatible with integrated electronics/optics facilities. To this end, the use of a silicon semiconductor wafer (on top of which the waveguide structure is grown) is an adequate choice. Finally, the physical differences (e.g., waveguide structure, temperature) between the signal and the reference arms (see Fig. 1) of the interferometer should be as small as possible. This can be obtained by placing both arms very close to each other and by using a waveguide configuration that is as homogeneous as possible across the wafer.

By choosing $\mathrm{Si}_{3} \mathrm{~N}_{4}\left(n_{\mathrm{f}}=2.0\right)$ for the waveguiding layer and $\mathrm{SiO}_{2}\left(n_{\mathrm{s}}=1.457\right)$ grown on top of an $\mathrm{Si}$ wafer for the substrate, a good compromise can be found. The refractive-index difference between the substrate and the waveguiding layer is then relatively high. Moreover, $\mathrm{Si}_{3} \mathrm{~N}_{4}$ layers, fabricated using low pressure chemical vapour deposition (LPCVD), make very homogeneous waveguides with low attenuation losses [6]. The thickness of the $\mathrm{Si}_{3} \mathrm{~N}_{4}$ layer should be $\approx 0.1 \mu \mathrm{m}$ in order to fulfil the condition given in eqn. (4b).

It then follows $[3,5]$, with $d_{\mathrm{f}}=95 \mathrm{~nm}$ :

$$
\begin{aligned}
\left(\frac{\partial N}{\partial d_{f}^{\prime}}\right)_{n_{f}^{\prime}} & =1.1 \times 10^{-3}\left(n_{\mathrm{f}}^{\prime 2}-n_{\mathrm{c}}^{2}\right) \\
& \cong 30 \times 10^{-4}\left(n_{\mathrm{f}}^{\prime}-n_{\mathrm{c}}\right) \mathrm{nm}^{-1}
\end{aligned}
$$

For the refractive index $n_{c}$ we take 1.33 (water). For the protein refractive index $n_{\mathrm{f}}^{\prime}$ the value 1.45 is often taken $[7,13]$, resulting in $\left(\partial N / \partial d_{f}^{\prime}\right) \approx 3.8 \times 10^{-4} \mathrm{~nm}^{-1}$. Equation (5a) shows that this sensitivity is strongly dependent on the refractive-index difference between $n_{\mathrm{c}}$ and $n_{f}^{\prime}$; and thus on $n_{f}^{\prime}$.

With $L=1 \mathrm{~cm}$ and $\lambda_{0}=632.8 \mathrm{~nm}$, it follows from eqn. (lb) that

$$
\Delta \phi \approx 6 \Delta d_{\mathrm{f}}^{\prime}(2 \pi)\left(d_{\mathrm{f}}^{\prime} \text { in } \mathrm{nm}\right)
$$

The dependence of the bulk refractive-index sensitivity $\partial N / \partial n_{\mathrm{c}}$ on the waveguide configuration is similar to that of $\partial N / \partial d_{\mathrm{f}}^{\prime}$ (see eqn. (3)), so it is also approximately at a maximum. Its value, with $n_{\mathrm{c}}=1.33$, is $\partial N / \partial n_{\mathrm{c}}=$ 0.17 , resulting in a corresponding phase change of

$$
\Delta \phi \approx 2.7 \times 10^{3}\left(\Delta n_{\mathrm{c}}\right)(2 \pi)
$$

The use of the materials $\mathrm{SiO}_{2}$ and $\mathrm{Si}_{3} \mathrm{~N}_{4}$ is also advantageous in another respect: both materials can be thoroughly cleaned without damage using standard cleaning techniques. The waveguide structure can thus be used several times without losing sensitivity. We usually cleaned the surfaces by soaking in neat $\mathrm{HNO}_{3}$ at room temperature and in $70 \% \mathrm{HNO}_{3}$ at $90{ }^{\circ} \mathrm{C}$. In this context it is desirable to provide the waveguide structure with grating couplers in order to prevent wafer damage by the prism-coupling technique that can also be used to couple light in and out of the waveguide.

In the next Section, we shall present the structure used in our experiments.

\section{Waveguide structure}

As the chosen waveguide configuration largely determines the manufacturing technology, we shall first comment on the general approach that we followed in designing a waveguide structure. Of course the most satisfactory option would be a totally integrated planar or channel waveguide structure. However, because of the difficulties in manufacturing fully integrated components required for light handling and signal processing, we have chosen a hybrid approach (see Section 4).

We use the waveguide structure shown in Fig. 3. It can be seen that the structure consists of a substrate on top of which a waveguide is fabricated. The complete waveguide is optically and chemically isolated from its surroundings, with two exceptions. The first consists of two areas where gratings are etched to provide optical

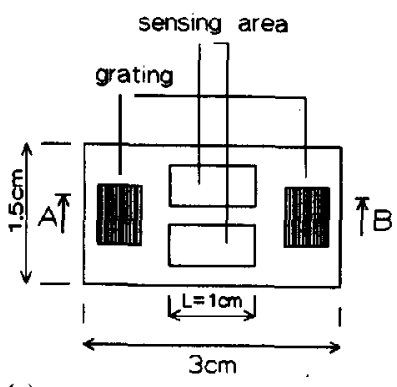

(a)

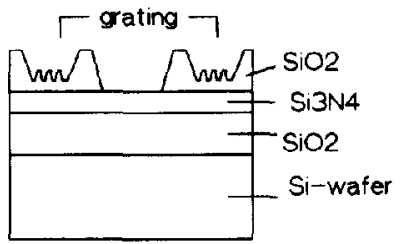

(b)

Fig. 3. The waveguide structure used: (a) top view; (b) cross section of the waveguide structure through the line A-B. The gratings are used to couple light in and out of the waveguiding $\mathrm{Si}_{3} \mathrm{~N}_{4}$ layer. Between the gratings and the sensing areas a cuvette can be pressed to the waveguide. Here an $\mathrm{SiO}_{2}$ layer $\approx 1.5 \mu \mathrm{m}$ thick provides optical isolation from the $\mathrm{Si}_{3} \mathrm{~N}_{4}$ layer. 
in- and outcoupling of the waveguide modes, respectively. The second exception consists of two sensing areas where surface adsorption takes place within the optically probed evanescent volume.

The thickness of the $\mathrm{Si}_{3} \mathrm{~N}_{4}$ layer was chosen to be $\cong 95 \mathrm{~nm}$, in order to facilitate efficient coupling of laser light using grating couplers. The interaction length $L$ is chosen to be $1 \mathrm{~cm}$, as a compromise between sensitivity and ease in handling the waveguide configuration.

The waveguide structure is fabricated using standard techniques. We start with a three inch wafer, onto which we thermally grow a $\mathrm{SiO}_{2}$ layer at $1150^{\circ} \mathrm{C}$. The thickness of this layer is approximately $1 \mu \mathrm{m}$ and it has a refractive index in 1.457. These data were measured with an ellipsometer (Applied Materials Ellipsometer II). The $\mathrm{SiO}_{2}$ layer acts as the substrate, on top of which the actual waveguiding layer of $\mathrm{Si}_{3} \mathrm{~N}_{4}$ is deposited by LPCVD at $800^{\circ} \mathrm{C}$. This $\mathrm{Si}_{3} \mathrm{~N}_{4}$ layer has a thickness $\approx 125 \mathrm{~nm}$, which is decreased to $95 \pm 1 \mathrm{~nm}$ by controlled wet chemical etching with an $\mathrm{HF}: \mathrm{NH}_{4} \mathrm{~F}$ (1:7) solution with known etching rate. The layer thickness and refractive index are measured both with the ellipsometer and the prism-coupling method [8].

On top of the $\mathrm{Si}_{3} \mathrm{~N}_{4}$ layer a protective $\mathrm{SiO}_{2}$ layer $\approx 1.5 \mu \mathrm{m}$ thick is grown at $300{ }^{\circ} \mathrm{C}$, using plasma-enhanced chemical vapour deposition (PECVD). This $\mathrm{SiO}_{2}$ layer is etched $\approx 250$ times faster than the $\mathrm{Si}_{3} \mathrm{~N}_{4}$ layer in the $\mathrm{HF}: \mathrm{NH}_{4} \mathrm{~F}$ solution mentioned above, providing an etch stop at the $\mathrm{Si}_{3} \mathrm{~N}_{4}$ layer. The thickness of the $\mathrm{SiO}_{2}$ layer can be accurately measured with a surface profiler (Sloan Dektak 3030).

In this waveguide configuration, only a $\mathrm{TE}_{0}$ mode can propagate; the $\mathrm{SiO}_{2}$ substrate is too thin to prevent the $\mathrm{TM}_{0}$ mode from being damped in the $\mathrm{Si}$ wafer. With the layers we use, thicker substrate layers will cause cracks because of internal stress differences between the $\mathrm{SiO}_{2}$ and the $\mathrm{Si}_{3} \mathrm{~N}_{4}$ layers.

The sensing areas are fabricated as follows: the protective $\mathrm{SiO}_{2}$ layer is completely removed (see Fig. 3) in two rectangles with a width of $3 \mathrm{~mm}$ and a length of $1.0 \mathrm{~cm}$ (interaction length). This is done by using standard photolithography on a positive photoresist layer (S1400-31 Shipley Microposit), followed by wet chemical etching using an $\mathrm{HF} / \mathrm{NH}_{4} \mathrm{~F}$ solution. The result is a tapered $\mathrm{SiO}_{2}$ layer with a taper angle of $\approx 10^{\circ}$, see Fig. 4. The resulting smooth gradient in cover-layer thickness considerably reduces light-scattering losses in this waveguide region.

In the same way, two rectangular regions with a remaining $\mathrm{SiO}_{2}$ thickness of $500 \mathrm{~nm}$ are fabricated, in which the in- and outcoupling gratings that are used to couple light into and out of the waveguide are located. The gratings are available on a chromium mask and have a grating constant of $2 \mu \mathrm{m}$ and $4 \mu \mathrm{m}$ for the in- and outcoupling gratings, respectively. The gratings are first

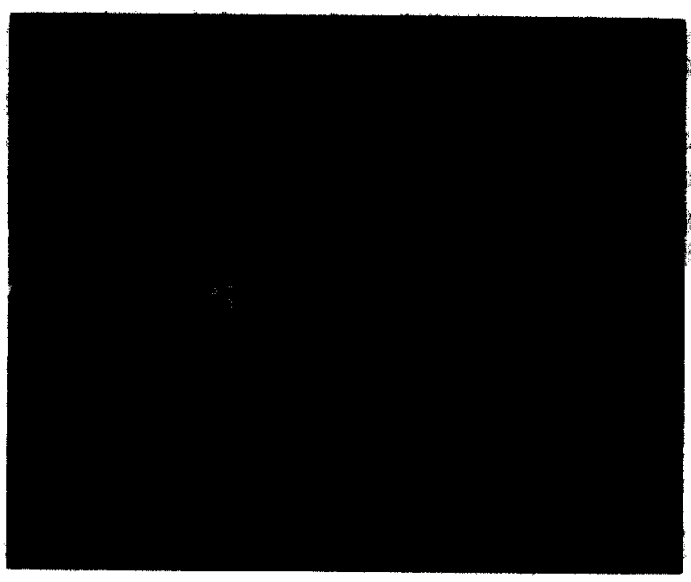

Fig. 4. SEM photograph of the cross section of the tapered transition between waveguide with and without $\mathrm{SiO}_{2}$ layer. The taper angle is $\approx 10^{\circ}$, resulting from a wet chemical etching technique.

fabricated in an aluminium layer (thickness $\approx 70 \mathrm{~nm}$ ), which is evaporated onto the waveguide structure in a vacuum chamber (evaporation pressure $3 \times 10^{-6}$ Torr). By using the reflective aluminium layer, the underlying layers are shielded, so the photolithography can be done very reproducibly with a high resolution [9]. The gratings are wet chemically etched into the aluminium layer using a standard combination of phosphoric, acetic and nitric acids ('aluminium etching liquid'). Then they are duplicated in the PECVD-oxide layer using reactive ion etching (RIE), after which the aluminium layer is removed using the mentioned etching liquid.

The grating efficiency is not only dependent on the underlying waveguide structure, but also on the grating depth and the grating profile [10]. In order to obtain a grating with reasonable efficiency (of the order of 1 $10 \%$ ), which is not sharply dependent upon the grating depth and profile, the grating should be separated from the waveguiding $\mathrm{Si}_{3} \mathrm{~N}_{4}$ layer by a thin remaining oxide layer. With our waveguide configuration, this is obtained by fabricating gratings with a grating depth of $\approx 300 \mathrm{~nm}$ in the region with the $500 \mathrm{~nm}$ thickness oxide layer. Here, the thickness of the $\mathrm{Si}_{3} \mathrm{~N}_{4}$ layer should not exceed $100 \mathrm{~nm}$, otherwise the efficiency drastically decreases. The choice of $\approx 95 \mathrm{~nm}$ also satisfies the sensitivity condition (eqn. 4(b)). An SEM photograph of the cross section of the waveguide in the grating region is shown in Fig. 5. Here the layered structure and the almost rectangular cross section of the grating, which results from the RIE etching process, are clearly visible.

Out of a three inch wafer, we can now make three of these complete structures, without any attempt to optimize this procedure. Finally, the structures are sawn out of the wafer, resulting in pieces of wafer of $\approx 3 \mathrm{~cm}$ length and $\approx 1.5 \mathrm{~cm}$ width (Fig. 6 ). 


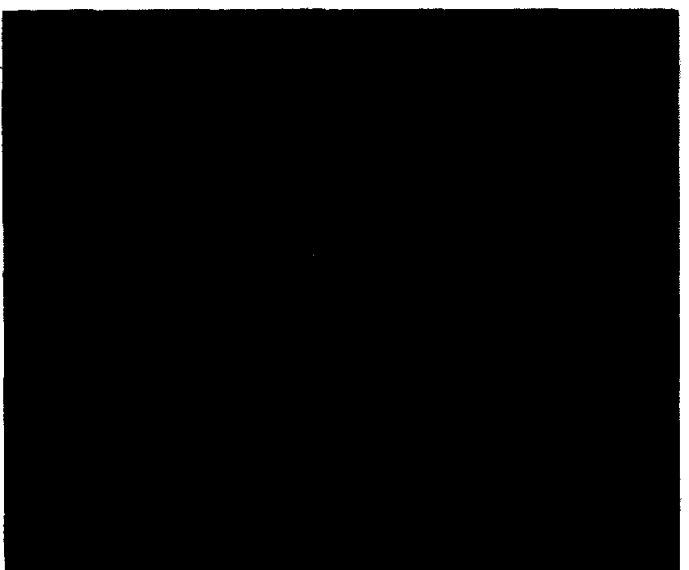

Fig. 5. SEM photograph of the cross section of the waveguide configuration in the grating region $(2 \mu \mathrm{m}$ grating constant), clearly indicating the layered structure and the almost rectangular grating profile caused by the RIE etching process.

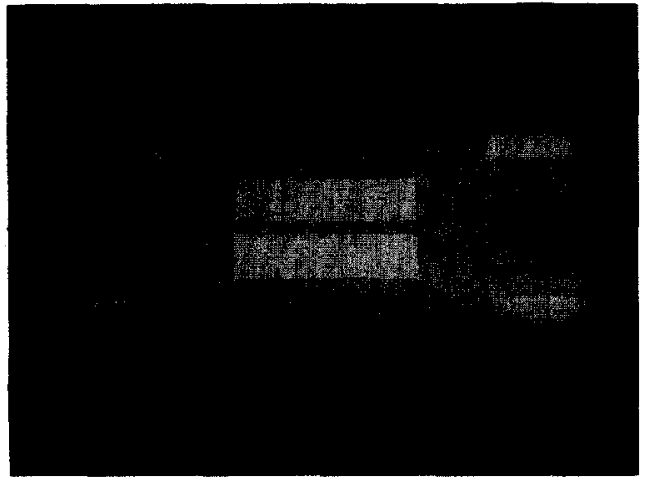

Fig. 6. Photograph of the waveguide structure used in the experiments, with a length of $3 \mathrm{~cm}$ and a width of $1.5 \mathrm{~cm}$.

These pieces are easily cleanable using strong acids without damaging the waveguide structures, which can be an important advantage because this makes the sensor reusable.

\section{Mach-Zehnder interferometer configuration}

The waveguide structure is placed in an interferometric set-up, which is mounted on a quartz block (not shown) in order to avoid stability problems.

As shown in Fig. 7, a laser beam ( $\mathrm{He}-\mathrm{Ne}, 633 \mathrm{~nm}$ ) is split into two parallel beams with the first cube beamsplitter. The distance between the two beams is $4 \mathrm{~mm}$. Both beams are coupled into and out of the waveguide structure using the two grating couplers described before. A second beamsplitter, at a distance of $4 \mathrm{~cm}$ from the first one, is used to recombine both beams, resulting in two interference patterns. A fixed

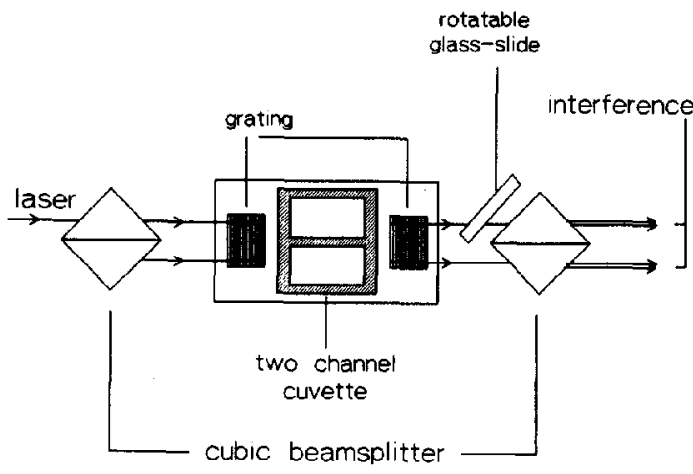

Fig. 7. Set-up for the waveguide interferometer.

(not shown in the Figure) and a rotatable glass slide (thickness $\approx 1 \mathrm{~mm}$ ) are placed in the two respective arms of the interferometer. The rotatable glass slide is mounted on a galvanometer optical scanner (General Scanning). Between the two grating couplers, a twochannel PTFE cuvette ( $0.8 \mathrm{ml}$ content) is pressed to the waveguide structure on top of the protective $\mathrm{SiO}_{2}$ layer, with a silicon rubber packing in between. By adjusting the waveguide-cuvette combination, two interference patterns are obtained, which are mutually out of phase [11].

The intensities of both interference patterns are measured with photodiodes. The interferometer is actively coupled back onto the quadrature points [12] of the two interference patterns by regulating the difference between the two intensities to zero. This is done by rotating the glass slide using a feedback loop. The regulation current is used as the signal, providing a linearized phase change as the interferometer output, a constant (maximal) sensitivity and a decreased intensity dependence.

The minimal phase change that can be measured is approximately $\left(1 \times 10^{-4}\right) 2 \pi$. However, there is some phase drift (probably caused by a temperature dependence of the galvanometer optical scanner) determining the measuring resolution. Without waveguide and cuvette, this drift is $\approx\left(2 \times 10^{-3}\right) 2 \pi$ per hour.

\section{Results}

\subsection{Coating of the waveguide surface}

In the immunoreaction experiments, we first check the sensitivity of the waveguide with bulk refractiveindex steps. Hereto both channels $(13 \mathrm{~mm}$ length $x$ $5.5 \mathrm{~mm}$ width $\times 14 \mathrm{~mm}$ height) of the cuvette are first filled with $0.8 \mathrm{ml}$ of pure water. After a stabilization period, a repeated substitution of half of the cuvette content by phosphate buffer solution (PBS) $(\mathrm{pH}=7.35$, 


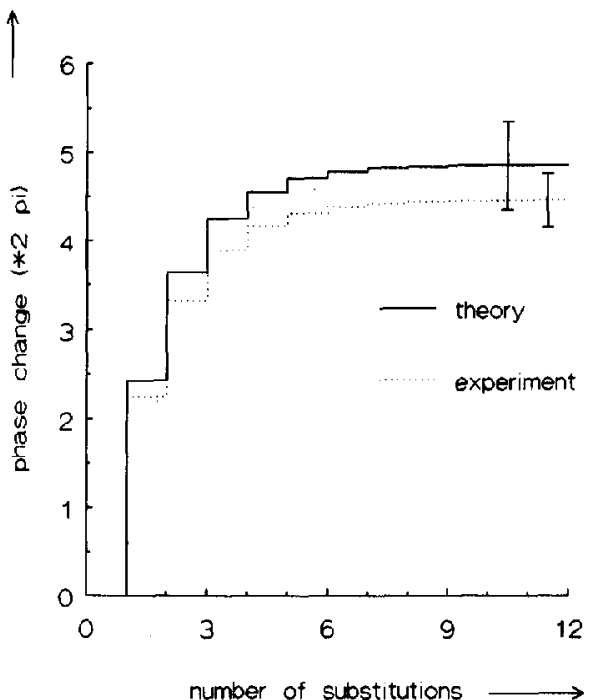

Fig. 8. The characteristic stepwise water-PBS refractive-index transition ( $\Delta n \approx 0.0018$, measured with an Abbe refractometer) in one of the channels.

osmolarity $=290 \mathrm{mOsmol}$.) in the measuring channel follows. This leads to a step-response curve. The result of such a measurement is shown in Fig. 8 .

As can be seen from this Figure, the resulting measured maximal phase change is $(4.5 \pm 0.3) 2 \pi$. The refractive index difference between the two liquids is $(1.8 \pm 0.2) \times 10^{-3}$, as was measured with an Abbe refractometer. The expected phase change with the waveguide structure as described in Section 2 is then $(4.8 \pm 0.5) 2 \pi$ (see Fig. 8).

After this sensitivity check the coating procedure is started. In order to be able to monitor this procedure, the two channels are treated successively.

As a first step in the coating procedure, an antibody containing PBS solution is applied to one channel. The use of the antibody anti-human chorionic gonadotropin ( $\alpha \mathrm{hCG}$, molecular weight $\approx 150 \mathrm{kDa}$ ) in a concentration of $5 \mu \mathrm{g} \mathrm{ml}^{-1}$ results in an adsorption process as shown in Fig. 9(a). Once a phase change of $\approx 10 \times 2 \pi$ is obtained, the antibody-containing solution is removed and the channel is washed thoroughly by repeated flushing with PBS. Subsequently, the same procedure is applied to the other channel.

Saturation of the adsorption results in a phase change of $\approx 35 \times 2 \pi$. Assuming an antibody refractive index $n_{f}^{\prime}=1.45$ [13], we calculate with eqn. (5b) an average layer thickness $\approx 6 \mathrm{~nm}$. This is in reasonable agreement with a monolayer coverage of a $150 \mathrm{kDa}$ mol. wt. protein $[7,13]$. Note, however, that this number is strongly dependent on the assumed value of the refractive index: if $n_{f}^{\prime}=1.50$ is assumed, a thickness $\approx 4 \mathrm{~nm}$ is calculated.
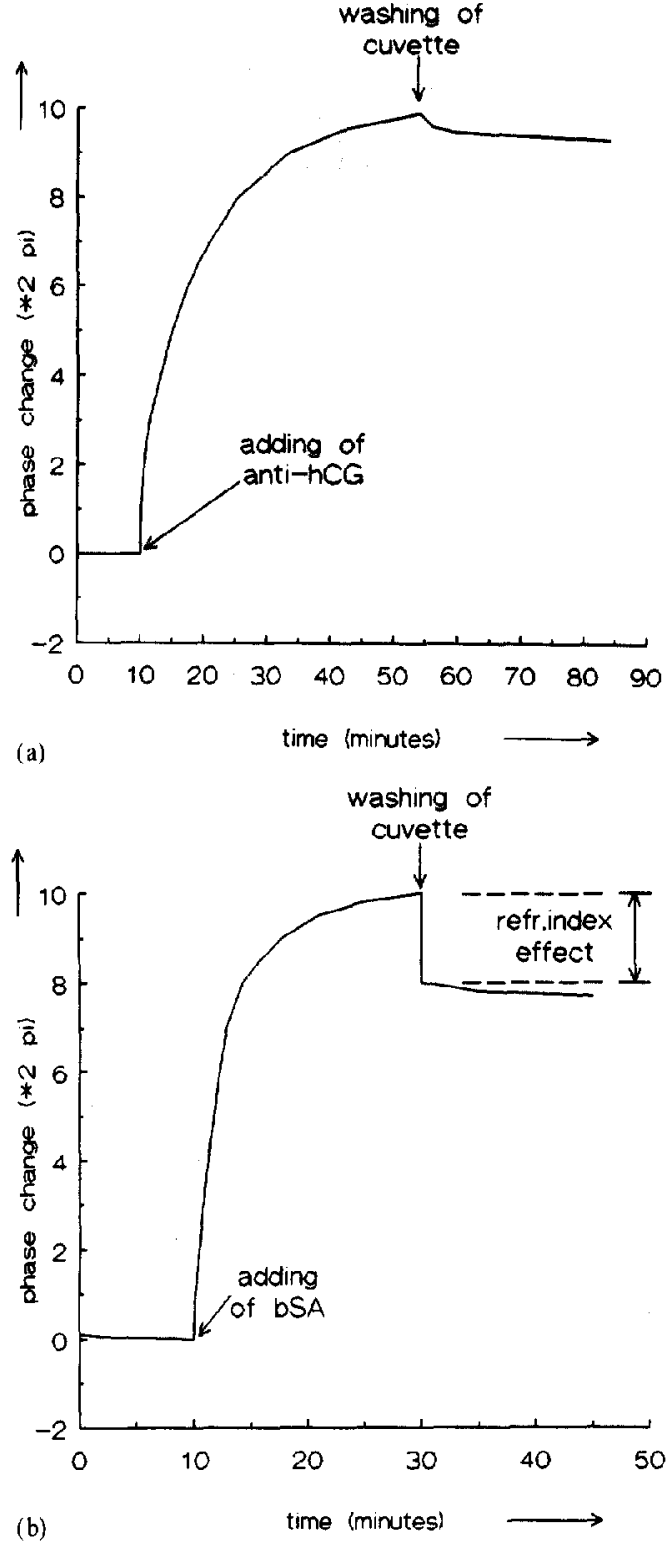

Fig. 9. Phase change caused by (a) the adsorption of $3 \times 10^{-8} \mathrm{M}$ ahCG (receptor molecules) and (b) $2 \times 10^{-4} \mathrm{M}$ bSA (block procedure) as a function of time.

We found, however, that a complete monolayer coverage of the waveguide surface dramatically reduces the immunoreaction activity [14], so we decided to coat the surface with about a quarter of a monolayer of $\alpha$ hCG. Furthermore, we found [14] that the hydrophobicity of the waveguide surface strongly determines the immunoreactivity of the adsorbed antibody molecules: for optimal immunoresponse the waveguide surface should be hydrophobic. This is realized by treating the cleaned 
(hydrophilic) waveguide with evaporated hexamethyldisilane (HMDS) before the experiments. This results in a monolayer of hydrophobic methyl groups on the surface.

The next step in the coating procedure consists of filling the measurement channel with a PBS buffer containing a high concentration of bovine serum albu$\min (\mathrm{bSA})\left(5 \mathrm{mg} \mathrm{ml}^{-1}\right.$, mol. wt. $\left.\approx 50 \mathrm{kDa}\right)$. This is done in order to prevent non-specific adsorption [15]. The bSA adsorption results in a further phase change of $\approx 10 \times 2 \pi$ (see Fig. 9(b)). After $20 \mathrm{~min}$, the channel is thoroughly washed again with PBS. It was seen that part of the phase change was caused by the bulk refractiveindex change due to the addition of the highly concentrated bSA solution. After the washing procedure a net phase change $\approx 7 \times 2 \pi$ remains. Finally, the other channel undergoes the same procedure.

\subsection{Immunosensing}

After the successive treatment of the two channels we wait until the drift is $\approx\left(1 \times 10^{-2}\right) 2 \pi$ per hour. This value is obtained after a stabilization period of approximately one hour. Although corrections can be made for the drift, the experimental situation is such that $\left(1 \times 10^{-2}\right) 2 \pi$ is regarded as the minimal phase change that can be unambiguously detected in the case of the immuno experiments described below.

In the immuno experiments half of the cuvette content of the reference channel is replaced by PBS and half of the cuvette content of the measuring channel by a hCG-containing PBS solution. The solution is not stirred. Once equilibrium is reached, the procedure is repeated with an increased concentration of hCG. After application of a concentration of $1 \times 10^{-9} \mathrm{M}$ hCG, a highly concentrated $\left(2 \times 10^{-8} \mathrm{M}\right)$ solution of a different antigen, hSA, is added to check the specificity of the reaction.

The time dependence of the phase change that results from successive additions is shown in Fig. 10(a). Here, the phase change is plotted on a logarithmic scale. The concentration dependence of the maximal phase changes is given in Fig. 10(b), with the hCG concentration plotted on a logarithmic scale.

The lowest detectable concentration is $5 \times 10^{-11} \mathrm{M}$ hCG $\left(2.5 \mathrm{ng} \mathrm{ml}^{-1}\right)$, causing a phase change of $\left(1 \times 10^{-2}\right) 2 \pi$ in approximately $20 \mathrm{~min}$. Here we have corrected for the drift. This is equivalent to an average adlayer growth of $1 \times 10^{-3} \mathrm{~nm}$. Note that this number was calculated assuming a constant $n_{\mathrm{f}}^{\prime}$. The immunoreaction signal continues at increasing concentration of hCG. Finally a saturation level is reached (Fig. 10(b)), corresponding to complete antigen coverage of all available active binding sites. This maximal value is $\approx 1.4 \times 2 \pi$, which means, in view of the $10 \times 2 \pi$ phase change caused by the antibody adsorption, that
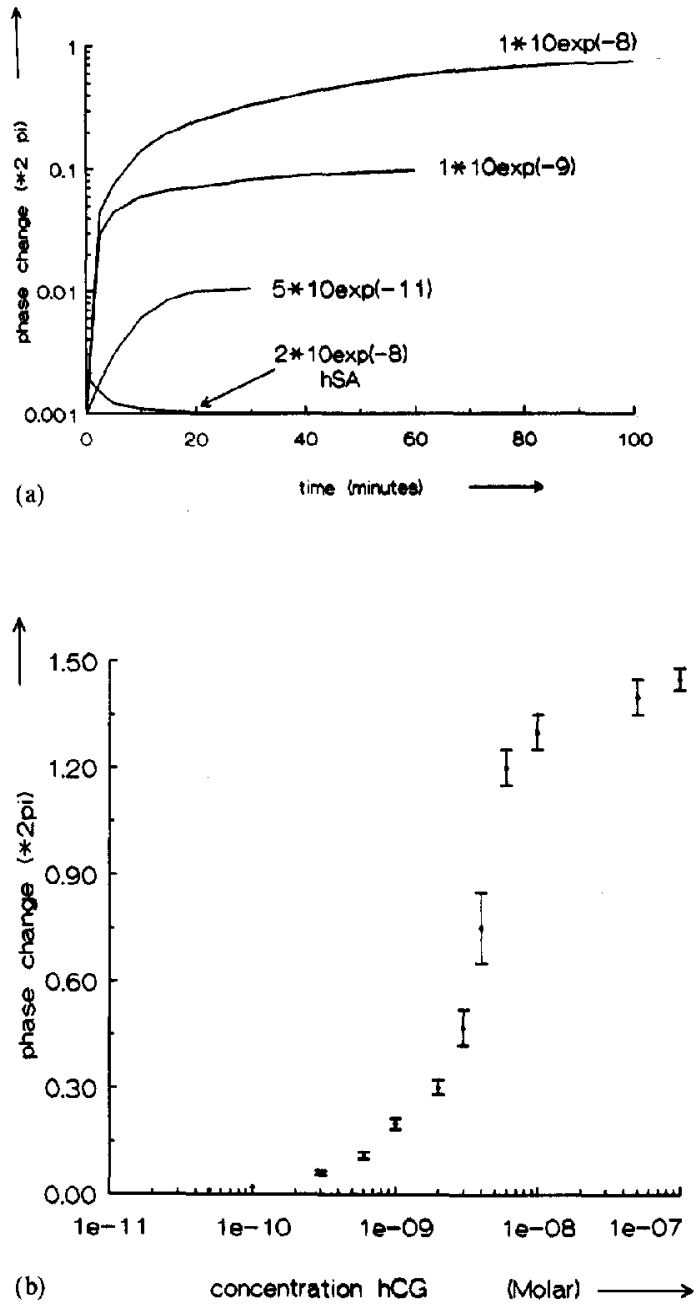

Fig. 10. The immunoreaction experiments. (a) The extra phase change caused by some immunoreactions as a function of time, induced by adding successive concentrations of antigen $\mathrm{hCG}$, with the concentration of the antigen as parameter. (b) The maximal phase change as a function of the concentration of hCG. The error bars represent the deviations from the average result, obtained by repeating the experiments.

only a fraction of the adsorbed $\alpha \mathrm{hCG}$ molecules can bind an antigen. From the concentration dependence of the hCG response the overall affinity constant can be determined. This topic will be treated elsewhere [14]. However, a rough estimate from Fig. 10(b) shows that the affinity constant is of the order of $10^{8} \mathrm{M}^{-1}$, as judged from the point of maximum slope of the Langmuir curve.

Finally, the absence of any positive response to a large hSA concentration clearly demonstrates that the obscrved $\alpha \mathrm{hCG}-\mathrm{hCG}$ reaction is specific. 


\section{Discussion and conclusions}

We have demonstrated that a planar waveguide Mach-Zehnder interferometer is capable of detecting extremely thin layers of adsorbed molecules. The combination of optical sensitivity, provided by proper design of the waveguide configuration, and chemical affinity, because of the presence of immobilized active antibodies with high specific affinity, results in a device that can quantitatively and selectively detect a bulk concentration of $5 \times 10^{-11} \mathrm{M}$ hCG. This sensitivity is at least one order of magnitude better than that of other previously described evanescent-field sensors, based on surface plasmon resonance [16] or grating couplers [17]. The present performance is by no means optimized yet: from the measured maximum $\alpha \mathrm{hCG}$ :hCG coverage ratio it can be inferred that the antibody-coating procedure can be significantly improved. We expect that an even more significant improvement can be obtained by circumventing the long measuring times caused by the diffusion-controlled kinetics of the immunoreaction. This will result in a reduced overall drift of the device during the course of the experiment. Implementation of a flow-injection analysis (FIA) system in the present device seems promising in this respect.

It should be noted that the device in its present form is only capable of recording the kinetics of a surface affinity reaction, because the present device only measures relative phases. If one is interested in performing endpoint titrations, such as is usual in conventional ELISA experiments, more complicated detection schemes are necessary [18].

In this communication we have focused upon the detection of very thin layers bound to the waveguide surface. Of course, a similar device can be employed to measure bulk refractive-index changes down to $\Delta n \approx 4 \times 10^{-6}$, corresponding to a phase change $\approx\left(1 \times 10^{-2}\right) 2 \pi$. However, in order to avoid spurious response due to the adsorption of molecular layers, it is then better to use a waveguide configuration that is insensitive to adsorption, e.g., as indicated by Lukosz et al. [19]. In particular for this last application it can be an advantage that the device can withstand severe cleaning conditions without any loss of optical sensitivity.

\section{Acknowledgements}

These investigations in the program of the Foundation for Fundamental Research on Matter (FOM) have been supported (partly) by the Netherlands Technology Foundation (STW).

The gratings were fabricated with the aid of a grating mask. For the use of this mask, we would like to thank Dr P. V. Lambeck, Electronics Department, University of Twente, and Dr A. H. Verbruggen, Center for Submicron Technology (CST), University of Delft, The Netherlands.

The proteins $\alpha \mathrm{hCG}$ and $\mathrm{hCG}$ were kind gifts from $\mathrm{Dr}$ K. M. P. Kamps, Organon International, Oss, The Netherlands.

\section{References}

I J. F. Place, R. M. Sutherland and C. Dahne, Opto-electronic immunosensors: a review of optical immunaassay at continuous surfaces, Biosensors, 1 (1985) 321-353.

2 R. E. Dessy, Waveguides as chemical sensors, Anal. Chem., 61 (1989) 1079a-1094a

3 R. G. Heideman, R. P. H. Kooyman, J. Greve and B. S. F Altenburg, A simple interferometer for evanescent field refractive index sensing as a feasibility study for an immunosensor, Appl. Opt., 30 (1991) 1474-1479.

4 R. G. Heideman, R. P. H. Kooyman and J. Greve, Development of an optical waveguide interferometric immunosensor, Sensors and Actuators B, 4 (1991) 297-299.

$5 \mathrm{~K}$. Tiefentahler and W. Lukosz, Sensitivity of grating couplers as integrated-optical chemical sensors, J. Opt. Soc. Am. B., 6 (1989) 209-220

$6 \mathrm{~W}$. Stitius and W. Streifer, Silicon nitride films on silicon for optical waveguides, Appl. Opt., 16 (1977) 3218-3222.

7 H. E. de Bruijn, B. S. F. Altenburg, R. P. H. Kooyman and J. Greve, Determination of thickness and dielectric constant of thin transparent dielectric layers using surface plasmon resonance, Opt. Commun., 82 (1991) 425-432.

8 P. K. Tien, R. Ulrich and R. J. Martin, Modes of propagating light waves in thin deposited semiconductor films, Appl. Phys. Lett., 14 (1969) 291-294.

$9 \mathrm{II}$. J. Lee, Techniques for fabricating Bragg reflectors on $\mathrm{SiO}_{2}-\mathrm{Si}_{3} \mathrm{~N}_{4}-\mathrm{SiO}_{2}$ rib waveguides on $\mathrm{Si}$, Appl. Opt., $27(1988)$ $1199-1202$

10 T. Tamir and S. T. Peng, Analysis and design of grating couplers, Appl. Phys., 14 (1977) 235-254.

$11 \mathrm{~V}$. Degiorgio, Phase shift between the transmitted and the reflected optical fields of a semireflecting lossless mirror is $\pi / 2$, Am. J. Phys., 48 (1980) 81-82.

12 D. Vilkomerson, Measuring pulsed picometer-displacement vibrations by optical interferometry, Appl. Phys. Lett, 29 (1976) 183-185

13 P. A. Cuypers, J. W. Corsel, M, P. Janssen, J. M. M. P. Kop, W. Th. Hermens and $\mathrm{H}$. C. Henker, The adsorption of prothrombin to phosphatidylserine multilayers quantitated by ellipsometry, J. Biol. Chem., 258 (1983) 2426-2431.

14 R. G. Heideman, R. P. H. Kooyman and J. Greve, Immunoreactivity of adsorbed anti human chorionic gonadotropin $(\alpha h C G)$ studied with an optical waveguide interferometric sensor, Biosensors Bioelectron., submitted for publication.

15 R. H. Hardy, in D. M. Weir (ed.), Handbook of Experimental Immunology, Vol. 1, Blackwell, Oxford, 1986, Ch. 13.

16 R. P. H. Kooyman, H. E. de Bruijn, R. G. Eenink and J. Greve, Surface plasmon as a bioanalytical tool, J. Mol. Struct., 218 (1990) 345-350.

17 W. Lukosz, D. Clerc and Ph. M. Nellen, Input and output grating couplers as integrated optical biosensors, Sensors and Actuators A, 25-27 (1991) 181-184.

18 R. M. Nakamura, A. Voller and D. E. Bidwell, in D. M. Weir (ed.), Handbook of Experimental Immunology, Vol. 1, Blackwell, Oxford, 1986, Ch. 27. 
19 W. Lukosz and Ch. Stamm, Integrated optical interferometer as relative humidity sensor and differential refractometer, Sensors and Actuators A, 25-27 (1991) 185-188.

\section{Biographies}

Rene Heideman was born in Goor, The Netherlands in April 1965. He received his M.S. degree in applied physics from the University of Twente, The Netherlands, in August 1988. He is presently working towards his $\mathrm{Ph} . \mathrm{D}$. degree by developing biochemical sensors based on planar and fiber optical waveguides.

Rob Kooyman received his M.S. degree in physics at Leiden University in 1975. From 1975 to 1980 he worked at the Agricultural University at Wageningen on a $\mathrm{Ph} . \mathrm{D}$. thesis devoted to molecular spectroscopy. At the University of Utrecht he developed spectro- scopic methods for use in membrane biophysics. Since 1985 he has been involved in biosensor research at the University of Twente.

Jan Greve was born in Koog aan de Zaan, The Netherlands, on October 24, 1939. He received his M.S. degree in experimental physics from the Free University, Amsterdam, The Netherlands, in 1967 and the Ph.D. degree from the same University in 1972.

Since 1967 he has been working in the field of biophysics, applying and developing optical and spectroscopic techniques for the research of biological macromoles, bacteriophages and cells. In 1980 he started a biophysics subgroup of the opto-electronics group in the Applied Physics Department of the University of Twente. The current research interests of the group are micro-Raman spectroscopy of biological macromolecules, cell characterization, mini laser Doppler flowmeters and biosensors. 